Commun. Fac Sci. Univ. Ank Ser. A1 Math. Stat.

Volume 69, Number 1, Pages 576-602 (2020)

DOI: $10.31801 /$ cfsuasmas. 606890

ISSN 1303-5991 E-ISSN 2618-6470

http://communications.science.ankara.edu.tr/index.php?series=A1

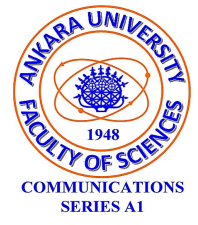

\title{
A STUDY ON COMPARISONS OF BAYESIAN AND CLASSICAL PARAMETER ESTIMATION METHODS FOR THE TWO-PARAMETER WEIBULL DISTRIBUTION
}

\author{
ASUMAN YILMAZ, MAHMUT KARA, AND HALIL AYDOGDU
}

\begin{abstract}
The main objective of this paper is to determine the best estimators of the shape and scale parameters of the two parameter Weibull distribution. Therefore, both classical and Bayesian approximation methods are considered. For parameter estimation of classical approximation methods maximum likelihood estimators (MLEs), modified maximum likelihood estimatorsI (MMLEs-I), modified maximum likelihood estimators -II (MMLEs-II), least square estimators (LSEs), weighted least square estimators (WLSEs), percentile estimators (PEs), moment estimators (MEs), L-moment estimators (LMEs) and TL- moment estimators (TLMEs) are used. Since the Bayesian estimators don't have the explicit form. There are Bayes estimators are obtained by using Lindley's and Tierney Kadane's approximation methods in this study. In Bayesian approximation, the choice of loss function and prior distribution is very important. Hence, Bayes estimators are given based on both the non- informative and informative prior distribution. Moreover, these estimators have been calculated under different symmetric and asymmetric loss functions. The performance of classical and Bayesian estimators are compared with respect to their biases and MSEs through a simulation study. Finally, a real data set taken from Turkish State Meteorological Service is analysed for better understanding of methods presented in this paper.
\end{abstract}

\section{INTRODUCTION}

Weibull distribution is one of the most popular among life-time distributions. The Weibull distribution was first proposed by W. Weibull who used it to model the distribution of the breaking strength of materials. The distribution has played major role in the reliability theory, see for example, 1 and $[2$. Also, the distribution has found wide applications in many areas of environmental sciences, and renewable energy [3, [4, [5] and [6] . In addition to these application areas, Weibull distribution

Received by the editors: August 20, 2019; Accepted: December 18, 2019.

2010 Mathematics Subject Classification. Primary 05C38, 15A15; Secondary 05A15, 15A18.

Key words and phrases. Bayes approximation, parameter estimation, new estimator, L- moment estimator, simulation study.

(C) 2020 Ankara University Communications Faculty of Sciences University of Ankara-Series A1 Mathematics and Statistics 
is now being used in a wide range of fields in medical, biological, and earth sciences. For details, see [7], [8] and [9] .

It is crucial to determine the best parameter estimation method for any probability function. There are various different estimation methods in the literature for estimating the parameters of the Weibull distribution. Notable among them are given as follows: In terms of classical parameter estimation methods, Trustrum and Jayatilaka [10] investigated the moment estimator, maximum likelihood estimator and least squares method based on the Monte Carlo simulation.Hung [11] and $\mathrm{Lu}$ et al.[12 discussed the properties of the weighted least square estimators and showed that weighted least squares estimators performed better than least squares estimators. Pobocikova and Sedliackova 13 compared the maximum likelihood estimators, moment estimators, least squares estimators and weighted least square estimators. Teimouri et al. [14 presented the maximum likelihood estimators, method of logarithm moment, percentile estimator, L- moment estimator, method of moment. Alizadeh et al. [15] considered estimation of the probability density function and cumulative density function.

In terms of Bayesian parameter estimation methods, Al Omari and Ibrahim [16] conducted a study on Bayesian survival estimator for Weibull distribution with censored data. Also, Guure et al. [17] provided the Bayesian estimation of two parameter Weibull distribution under three loss functions using extension of Jeffey's prior information. Pandey et. al [18, compared Bayesion estimator and maximum likelihood estimation of the scale parameter of the Weilbull distribution under linex loss function, with the assumption that the shape parameter is kwown. Similar work can be seen in [19, [20] .

The maximum likelihood estimators (MLEs) and the moment estimators (MEs) are the most well-known among parameter estimation methods. In this article, the least square error estimators (LSEs) and the weighted square error estimators (WLSEs), the percentile estimators (PEs),the L-moment estimators (LMEs), the TL-moment estimators (TLMEs), modified maximum likelihood estimators (MMLEI) are considered besides these methods. Moreover, we propose the modified maximum likelihood estimators-II (MMLE-II). Further, we compute Bayes estimators of the unknown parameters with informative prior and non-informative prior under squared error loss function (SELF), general entropy loss function (GELF), weighted square loss function (WSELF) and precautionary loss function (PLF). It is clear that Bayesian estimators cannot be found in explicit form. Therefore, in this paper, we consider the Lindley's and Tierney Kadane's procedures.

There are numerous studies for Weibull distribution in literature. But, as far as we know this, this is the first study which compares all these aforementioned estimation methods for choosing the best estimation method for the two- parameter Weibull distribution. The objective of this study is to estimate the parameters of the model from both classical and Bayesian viewpoint. Finally, a better estimation method is given for the distribution parameters. In the recent past, many 
researchers have compared various parameter estimation methods for estimating the parameters of the different distribution. See, for example, 21] for the generalized Rayleigh distribution, 22] for the Fréchet distribution, 23] for two parameter Maxwell distribution, 24] for generalized logistic distribution.

The rest of the paper is organized as follows: Weibull distribution is described in section 2 . In section 3 , some classical estimation methods are given to estimate the unknown parameters. In section 4, Bayes estimators of the unknown parameters are obtained by using Lindley's and Tierney Kadane's approximations. In Section 5, a simulation study is presented to evaluate the performances of the estimators with respect to their biases and mean square errors (MSE). Finally, a real life example taken from Turkish State Meteorological Service is given.

\section{Weibull Distribution}

The popularity of the Weibull distribution is attributable to the fact that it is commonly used to model different data types, such as wind speed, geothermal energy and finance.

The probability density function (PDF) and the cumulative density function $(\mathrm{CDF})$ of the two-parameter Weibull distribution with the shape parameter $\alpha$ and the scale parameter $\beta$ are given by:

$$
F(x ; \alpha, \beta)=1-\exp \left\{-\left(\frac{x}{\beta}\right)^{\alpha}\right\} 0<x<\infty ; \alpha>0, \beta>0
$$

and

$$
f(x ; \alpha, \beta)=\frac{\alpha}{\beta^{\alpha}} x^{\alpha-1} \exp \left\{-\left(\frac{x}{\beta}\right)^{\alpha}\right\}, 0<x<\infty .
$$

The mean and variance of the Weibull distribution are defined as follows:

$$
E(x)=\beta \Gamma\left(1+\frac{1}{\alpha}\right) \text { and } V(x)=\beta^{2}\left[\Gamma\left(1+\frac{2}{\alpha}\right)-\Gamma^{2}\left(1+\frac{1}{\alpha}\right)\right]
$$

respectively. Here, $\Gamma$ is the gamma function.

\section{The Methods for Parameter Estimation}

In this section, we presented the methods of classical estimation for the Weibull distribution used in this study.

3.1 Moment Estimators. The MEs are found by equating theoretical moments to corresponding sample moments as shown below:

$$
\beta \Gamma\left(1+\frac{1}{\alpha}\right)=\bar{X} \text { and } \beta^{2} \Gamma\left(1+\frac{2}{\alpha}\right)=\frac{\sum_{i=1}^{n} X_{i}^{2}}{n} .
$$


Then, by solving equation 3 the MEs of $\alpha$ and $\beta$ are found as

$$
\hat{\beta}=\frac{\bar{X}}{\Gamma\left(1+\frac{1}{\alpha}\right)} \text { and } \frac{\Gamma\left(1+\frac{2}{\hat{\alpha}}\right)}{\Gamma^{2}\left(1+\frac{2}{\hat{\alpha}}\right)}=\frac{\sum_{i=1}^{n} X_{i}^{2}}{n \bar{X}^{2}}
$$

respectively.

3.2 Maximum Likelihood Estimators. Let $X_{1}, X_{2}, \ldots, X_{n}$ be a random sample from Weibull distribution. The log-likelihood function is given by:

$$
\ln L=n \ln \alpha-n \alpha \ln \beta+(\alpha-1) \sum_{i=1}^{n} \ln x_{i}-\sum_{i=1}^{n}\left(\frac{x_{i}}{\beta}\right)^{\alpha} .
$$

By taking the partial derivative of 5 with respect to $\alpha$ and $\beta$, and equating them to zero, we obtain the following log-likelihood equations:

$$
\frac{\partial \ln L}{\alpha}=\frac{n}{\alpha}-n \ln \beta+\sum_{i=1}^{n} \ln x_{i}-\sum_{i=1}^{n}\left(\frac{x_{i}}{\beta}\right)^{a} \ln \frac{x_{i}}{\beta}=0
$$

and

$$
\frac{\partial \ln L}{\partial \beta}=\frac{n \alpha}{\beta}+\frac{\alpha \sum_{i=1}^{n} x_{i}^{\alpha}}{\beta^{\alpha+1}}=0 .
$$

Solutions of these likelihood equations are called as the MLEs of shape parameter $\alpha$ and scale parameter $\beta$, see for example 25], 26] . However, they do not give closed form expressions since they include nonlinear terms $g_{1}(x)=\ln x$ and $g_{2}(x)=x_{i}^{\alpha}$ in 6 and 7. Therefore, numerical methods are applied to solve the required equations. In this study, we apply the well-known Newton Rapson method to solve these equations.

3.3 Least Squares and Weighted Least Squares Estimators. The LSEs and WLSEs were originally suggested by Swain et al.27] to estimate the parameters of beta distributions. See, for example, Kundu and Ragab [21] and Alkasabeh and Ragab 24.

Let $X_{1}, \ldots, X_{n}$ is a random sample of size $n$ from a distribution function $G($.$) and$ $X_{i: n} ; i=1,2, \ldots, n$ denotes the ordered sample. The expected value and variance of $G\left(X_{i: n}\right)$ are easily obtained from the relation between the Beta and uniform distribution as

$$
E\left(G\left(X_{i: n}\right)\right)=\frac{i}{n+1} \text { and } \operatorname{Var}\left(G\left(X_{i: n}\right)\right)=\frac{i(n-i+1)}{(n+1)^{2}(n+2)} .
$$

Since $E\left(G\left(X_{i: n}\right)\right)=\frac{i}{n+1}, i=1,2, \ldots, n$, a regression model can be written as follows:

$$
G\left(X_{i: n}\right)=\frac{i}{n+1}+\varepsilon_{i}, i=1,2, \ldots, n .
$$


Then the LSEs of the unknown parameters can be obtained by minimizing the sum of squares of errors

$$
\sum_{i=1}^{n}\left(G\left(X_{i: n}\right)-\frac{i}{n+1}\right)^{2}
$$

with respect to unknown parameters. Therefore, the LSEs of the unknown parameters of Weibull distribution are found by minimizing

$$
\sum_{i=1}^{n}\left(1-\exp \left(-\left(x_{i: n} / \beta\right)^{\alpha}\right)\right)^{2}
$$

with respect to $\alpha$ and $\beta$. Since the variances of errors depend on $i$, the heteroscedasticity problem arises. This problem adversely affects the performance of the estimators. To overcome this problem, we use the method of weighted least squares. The weighted least squares estimators of the unknown parameters can be obtained by minimizing

$$
\sum_{i=1}^{n} W_{i}\left(G\left(X_{i: n}\right)-\frac{i}{n+1}\right)^{2}
$$

with respect to the unknown parameters. Therefore, the WLSEs of the unknown parameters of the two-parameter Weibull distribution are obtained by minimizing

$$
\sum_{i=1}^{n} w_{i}\left(1-\exp \left\{-\left(x_{i: n} / \beta\right)^{\alpha}\right\}\right)^{2}
$$

with respect to $\alpha$ and $\beta$. Where $W_{i}=\frac{(n+1)^{2}(n+2)}{i(n-i+1)}$.

3.4 The Percentile Estimators. The Percentile estimators (PEs) of $\alpha$ and $\beta$ are obtained by minimizing the function given below:

$$
\sum_{i=1}^{n}\left\{X_{i: n}-F^{-1}\left(\frac{i}{n+1}\right)\right\}^{2}
$$

with respect to unknown parameters [28], 29] . Here, $F^{-1}$ is the inverse distribution function and $X_{i: n}$ is ordered observations i.e. $X_{1: n}<X_{2: n}<\ldots<X_{n: n}$.

Then the PEs of the shape and scale parameters of the Weibull distribution are obtained by minimizing function

$$
\sum_{i=1}^{n}\left(X_{i: n}-\beta \ln \left(\frac{n+1}{n+1-i}\right)^{\frac{1}{\alpha}}\right)^{2}
$$

with respect to $\alpha$ and $\beta$.

3.5 L- Moment Estimators. The L- moment estimators (LMEs) was introduced by Hosking [30. These estimators have an estimation method based on linear combination of order statistics. The LMEs have lower sample variances and they are more robust outliers in data. In recently, a few authors have studied the Lmoment estimator for the Weibull distribution [14-31] . 
Let $X_{1}, X_{2}, \ldots, X_{n}$ be a random sample of size $n$ and $X_{1: n} \leq X_{2: n} \leq \ldots \leq X_{n: n}$ be the order random variables. Then the population L-moments and sample Lmoments are given as follows:

$$
\begin{gathered}
L_{k}=k^{-1} \sum_{j=0}^{k-1}(-1)^{j}\left(\begin{array}{c}
k-1 \\
j
\end{array}\right) E\left(X_{k-j: k}\right), k=1,2,3, \ldots, \\
l_{k}=\frac{1}{k\left(\begin{array}{l}
n \\
k
\end{array}\right)} \sum_{i=1}^{n} \sum_{j=0}^{k-1}(-1)^{j}\left(\begin{array}{c}
k-1 \\
j
\end{array}\right)\left(\begin{array}{c}
i-1 \\
k-j-1
\end{array}\right)\left(\begin{array}{c}
n-i \\
j
\end{array}\right) X_{i: n}, k=1,2,3, \ldots
\end{gathered}
$$

respectively. Here, $k$ is the number of the unknown parameters, $E\left(X_{i: n}\right)$ are the expected values of the order statistics and $n$ is sample size.

By using equations 14, the population L-moments of two-parameter Weibull distribution derived as

$$
L_{1}=\beta \Gamma\left(1+\frac{1}{\alpha}\right) \text { and } L_{2}=\beta \Gamma\left(1+\frac{1}{\alpha}\right)-\frac{\beta \Gamma\left(1+\frac{1}{\alpha}\right)}{2^{\frac{1}{\alpha}}} .
$$

The idea lying under $L$ moment estimators are the same as in the moment estimators. In other words, on equating the first two population moments to corresponding sample moments, the estimating equations are

$$
\beta \Gamma\left(1+\frac{1}{\alpha}\right)=l_{1} \text { and } \beta \Gamma\left(1+\frac{1}{\alpha}\right)-\frac{\beta \Gamma\left(1+\frac{1}{\alpha}\right)}{2^{\frac{1}{\alpha}}}=l_{2} .
$$

Then the LMEs of the parameters follow from 17 as

$$
\hat{\alpha}=\frac{\ln 2}{\ln \left(\frac{l_{1}}{l_{1}-l_{2}}\right)} \text { and } \hat{\beta}=\frac{l_{1}}{\Gamma\left(1+\frac{1}{\hat{\alpha}}\right)},
$$

respectively, where, $l_{1}=\bar{x}$ and $l_{2}=\frac{1}{n(n-1)} \sum(2 j-n-1) X_{j: n}$.

3.6 Trimmed L-Moments Estimators. Elamir and Seheult 32 proposed TLmoments as a robust generalization of L-moments. The TL-moments always exist even if the mean of the distribution does not exist, for example, the TL-moments exist for Cauchy distribution.

Let $X_{1}, X_{2}, \ldots, X_{n}$ be a random sample of size $n$ and $X_{1: n} \leq X_{2: n} \leq \ldots \leq X_{n: n}$ denote the corresponding order statistics. Elamir and Seheult [32] defined the kth the population and sample TL-moments

$$
\lambda_{k}^{(s, t)}=k^{-1} \sum_{j=0}^{k-1}(-1)^{j}\left(\begin{array}{c}
k-1 \\
j
\end{array}\right) E\left(X_{k+s-j: k+s+t}\right), k=1,2,3, \ldots, s, t=0,1,2, \ldots
$$

and

$$
l_{k}^{(s, t)}=\frac{1}{k\left(\begin{array}{c}
n \\
k+s+t
\end{array}\right)} \sum_{j=s}^{n-t} \sum_{i=0}^{k-1}(-1)^{i}\left(\begin{array}{c}
k-1 \\
i
\end{array}\right)\left(\begin{array}{c}
j-1 \\
k+s-i-1
\end{array}\right)\left(\begin{array}{c}
n-j \\
t+i
\end{array}\right) X_{j: n} k=1,2,3 \ldots
$$


respectively. It should be noted that TL-moments reduce to the L-moments when $s=t=0$. In this study, we focus on asymmetric cases where $s=0, t=1$. By putting $s=0$ and $t=1$ in equations 19 and 20 , we have

$$
\lambda_{k}^{(0,1)}=k^{-1} \sum_{j=0}^{k-1}(-1)^{j}\left(\begin{array}{c}
k-1 \\
j
\end{array}\right) E\left(X_{k-j: k+1}\right)
$$

and

$$
l_{k}^{(0,1)}=\frac{1}{k\left(\begin{array}{c}
n \\
k+t
\end{array}\right)} \sum_{j=0}^{n-1} \sum_{i=0}^{k-1}(-1)^{i}\left(\begin{array}{c}
k-1 \\
i
\end{array}\right)\left(\begin{array}{c}
j-1 \\
k-i-1
\end{array}\right)\left(\begin{array}{c}
n-j \\
i+1
\end{array}\right) X_{j: n} .
$$

The population TL-moments of the two-parameter Weibull distribution can be obtained from 21 as

$$
\lambda_{1}^{(0,1)}=\frac{\beta \Gamma\left(1+\frac{1}{\alpha}\right)}{2^{\frac{1}{\alpha}}} \text { and } \lambda_{2}^{(0,1)}=\frac{3 \beta \Gamma\left(1+\frac{1}{\alpha}\right)}{2^{\frac{1}{\alpha}}}-\frac{2 \beta \Gamma\left(1+\frac{1}{\alpha}\right)}{3^{\frac{1}{\alpha}}}
$$

The TLMEs are obtained by equating the first two sample TL-moments to the corresponding population TL-moments. Hence, the estimating equations are

$$
\frac{\beta \Gamma\left(1+\frac{1}{\alpha}\right)}{2^{\frac{1}{\alpha}}}=l_{1}^{(0,1)} \text { and } \frac{3 \beta \Gamma\left(1+\frac{1}{\alpha}\right)}{2^{\frac{1}{\alpha}}}-\frac{2 \beta \Gamma\left(1+\frac{1}{\alpha}\right)}{3^{\frac{1}{\alpha}}}=l_{2}^{(0,1)} .
$$

The solutions of these equations are the following TLMEs:

$$
\hat{\alpha}=\frac{\log \left(\frac{2}{3}\right)}{\log \left(\frac{3 l_{1}^{(0,1)}-2 l_{2}^{(0,1)}}{3 l_{1}^{(0,1)}}\right)} \text { and } \hat{\beta}=\frac{2^{1 / \alpha} l_{1}^{(0,1)}}{\Gamma\left(1+\frac{1}{\hat{\alpha}}\right)}
$$

where

$$
\left.l_{1}^{(0,1)}=\frac{2}{n(n-1)} \sum_{i=1}^{n-1}(n-j) X_{j: n} \text { and } l_{2}^{(0,1)}=\frac{3}{2 n(n-1)(n-2)} \sum_{j=1}^{n}(n-j)(3 j-n-1)\right) X_{j: n} .
$$

3.7 Modified Maximum Likelihood Estimators-I. Cohen and Whitten 33. recommend modifications of the MLEs for estimating the shape and scale parameters of the Weibull distribution. The MMLE-I of the shape parameter $\alpha$ and scale parameter $\beta$, say $\hat{\alpha}_{M M L E-I}$ and $\hat{\beta}_{M M L E-I}$ respectively, of the Weibull distribution is obtained by solving the following equations:

$$
\frac{-n X_{1: n}^{\alpha}}{\ln \left(\frac{n}{n+1}\right)}=\sum_{i=1}^{n} X_{i: n}^{\alpha} \text { and } \hat{\beta}_{M M L E-I}=\frac{1}{n}\left(\sum_{i=1}^{n} X_{i}^{\hat{\alpha}_{M M L E}}\right)^{\frac{1}{\hat{\alpha}_{M M L E-I}}}
$$

3.8 Modified Maximum Likelihood Estimators-II. We proposed modifications of the MLEs for estimating the unknown parameters of the Weibull distribution. Then, MMLE of the shape parameter $\alpha$, say $\hat{\alpha}_{M M L E-I I}$, is estimated by solving the following equation:

$$
\frac{\gamma+\frac{\ln \sum_{i=1}^{n} x_{i}^{\alpha}}{n}}{\alpha}=\frac{\sum_{i=1}^{n} \ln x_{i}}{n}
$$


where $\gamma \cong 0.57722$ is Euler constant.

Here, by inserting $\hat{\alpha}_{M M L E-I I}$ instead of $\hat{\alpha}$ into equation 7, MMLE of the scale parameter $\beta$, say $\hat{\beta}_{M M L E-I I}$ is obtained as

$$
\hat{\beta}_{M M L E-I I}=\left(\frac{1}{n} \sum_{i=1}^{n} x_{i}^{\hat{\alpha}_{M M L E-I I}}\right)^{\frac{1}{\bar{\alpha}_{M M L E-I I}}} .
$$

\section{Bayesian Analysis}

In this section, we consider the Bayesian estimation by using Lindley's and Tierney-Kadane's approximations under different loss function for estimating the unknown parameters of Weibull distribution. Bayesian analysis has many applications in statistical theory and analysis 34 . In Bayesian analysis the role of two factors are crucial. These are (i) the choice of the loss function (LF) and (ii) the choice of the prior distribution. For more details about the priors and loss functions, see [35, 36.

In this study, GELF, PLF, WSELF and SELF are considered and described as follows:

The SELF was proposed by Legendre [37] and Gauss [38] to developed least square theory. This loss function is commonly used and defined as

$$
L_{S E L F}=(\hat{\theta}-\theta)^{2},
$$

where $\theta$ is the parameter to be estimated by an estimator $\hat{\theta}$. The Bayes estimator under equation 28 is the posterior mean given by

$$
\hat{\theta}_{S E L F}=E(\theta \mid x) .
$$

This loss function is symmetrical in nature. It gives equal weight to both underestimation and over estimation. However, from a practical point of view, this is not always appropriate and realistic, see for example [39]. Hence, asymmetric loss functions would be more useful to develop Bayesian procedures.

Calabria and Pulcini [40] proposed general entropy loss function. It is one of the most popular asymmetrical loss functions.

The GELF is given by

$$
L_{G E L F}=\left(\frac{\hat{\theta}}{\theta}\right)^{k}-k \log \left(\frac{\hat{\theta}}{\theta}\right)-1, k \neq 0,
$$

where $\hat{\theta}$ is the estimator of $\theta$. $k$ reflects the magnitude and degree of symmetry. The Bayes estimator under equation 30 is given by

$$
\hat{\theta}_{G E L F}=\left[E\left(\theta^{-k} \mid x\right)\right]^{-\frac{1}{k}},
$$

provided $E_{\theta}\left(\theta^{-k} \mid x\right)$.

The PLF, which is proposed by Norstrom [41], is one of the asymmetric loss functions. This loss function approach is useful to derive conservative estimators since it approaches infinity near the origin and prevents underestimation. It is 
very useful when underestimation may lead to significant results [42]. The PLF is defined as

$$
L_{P L F}=\frac{(\theta-\hat{\theta})^{2}}{\hat{\theta}},
$$

where $\hat{\theta}$ is the estimator of $\theta$. The Bayes estimator of under equation 32 is given by

$$
\hat{\theta}_{P L F}=\sqrt{E\left(\theta^{2} \mid x\right)}
$$

provided $\sqrt{E\left(\theta^{2} \mid x\right)}$ exists and is finite.

WSELF is another useful asymmetric loss function. This function is a weighted version of SELF. More detail about this loss function can be found in [35] and [43]. The WSELF is defined as:

$$
L_{W S E L F}(\hat{\theta}, \theta)=\frac{(\theta-\hat{\theta})^{2}}{\hat{\theta}} .
$$

The Bayes estimator under WSELF is given by

$$
\hat{\theta}_{W S E L F}=\left[E\left(\theta^{-1} \mid x\right)\right]^{-1} .
$$

provided $E\left(\theta^{-1} \mid x\right)^{-1}$ exists and is finite.

The prior distribution summarizes the information about unknown parameter before the data is available. The prior distribution is then synthesized with the information in the data procedure the posterior distribution. In other words, analytically, combining the prior distribution and likelihood function results in the posterior distribution. The posterior distribution expresses what is known after seeing data. In the Bayesian analysis, all inferences are made from the posterior distribution [44].

The prior distribution has two forms: these are (i) "non-informative prior" and (ii) "informative prior" 45].

Here we assume that $\alpha$ and $\beta$ have two independent gamma prior distributions i.e. $\alpha \sim \operatorname{gamma}(a, b)$ and $\beta \sim \operatorname{gamma}(c, d)$ respectively. The gamma prior is very flexible and suitable. Thus, this paper considers two special cases of the gamma prior corresponding to $a=b=c=d=0$ and $a, b, c, d \geq 0(a, b, c, d$ are the hyper-parameters of the prior distribution). It should be mentioned that for $a=b=c=d=0$ the prior distribution is non-informative prior (NP) distribution. For $a, b, c, d \geq 0$, the prior distribution is referred to as the gamma prior (GP) distribution. Thus, the proposed prior for $\alpha$ and $\beta$ may be considered as

$$
v_{1}(\alpha) \propto \alpha^{a-1} e^{-b \alpha}, \alpha>0 \text { and } v_{2}(\beta) \propto \beta^{c-1} e^{-d \beta}, \beta>0 \text {. }
$$

The joint prior distribution $\alpha$ and $\beta$ is given as

$$
v(\alpha, \beta) \propto \alpha^{a-1} \beta^{c-1} e^{-d \beta-b \alpha}, \alpha, \beta, a, b, c, d \geq 0 .
$$


Based on the observations, the likelihood function becomes

$$
L(\alpha, \beta)=\alpha^{n} \beta^{-n \alpha} \prod_{i=1}^{n} X_{i}^{(\alpha-1)} e^{-\sum\left(\frac{X_{i}}{\beta}\right)^{\alpha}} .
$$

Combining 37 with 38 and using Bayes theorem, the joint posterior density of $\alpha$ and $\beta$ is

$$
p(\alpha, \beta \mid x)=K^{-1} \alpha^{n+a-1} \beta^{-n \alpha+c-1} \exp (-d \beta-b \alpha) \prod_{i=1}^{n} x_{i}^{\alpha-1} e^{\sum_{i=1}^{n}\left(\frac{x_{i}}{\beta}\right)^{\alpha}} .
$$

Here $K=\int_{0}^{\infty} \int_{0}^{\infty} \alpha^{n+a-1} \beta^{-n \alpha+c-1} \exp (-d \beta-b \alpha) \prod_{i=1}^{n} x_{i}^{\alpha-1} e^{\sum_{i=1}^{n}\left(\frac{x_{i}}{\beta}\right)^{\alpha}} d \alpha d \beta$.

It can be seen that the analytical solution of the Bayes estimators are not obtained. Hence, we use the Lindley's and Tierney-Kadane's approximation. These methods are described below.

4.1 Lindley's procedure. Lindley's [46] introduced an approximation method for the evaluation of the ratio of the two integrals. This procedure can be applied to compute the posterior expectation of the arbitrary function $u(\theta)$ as given by

$$
E(u(\theta) \mid x)=\frac{\int u(\theta) e^{L(\theta)+G(\theta)} d \theta}{\int e^{L(\theta)+G(\theta)} d \theta},
$$

where

$u(\theta)=$ a function of $\theta$ only,

$L(\theta)=$ Log-likelihood function,

$G(\theta)=\log$ of joint prior density function.

According to Lindley's approximation, the ratio of integral $E\{u(\theta) \mid x\}$ can be approximated asymptotically given below:

$E(u(\theta) \mid x) \approx\left[u+\frac{1}{2} \sum_{i} \sum_{j}\left(u_{i j}+2 u_{i} \rho_{i}\right) \sigma_{i j}+\frac{1}{2} \sum_{i} \sum_{j} \sum_{k} \sum_{l} L_{i j k} \sigma_{i j} \sigma_{k l} u_{l}\right]+O\left(1 / n^{2}\right)$.

Here, $i ; j ; k ; l=1,2, \ldots, n ; \theta=\left(\theta_{1}, \theta_{2}, \ldots, \theta_{m}\right), u_{i}=\frac{\partial u(\theta)}{\partial \theta_{i}}, u_{i j}=\frac{\partial u(\theta)}{\partial \theta_{i} \partial \theta_{j}}, L_{i j k}=$ $\frac{\partial L(\theta)}{\partial \theta_{i} \partial \theta_{j} \partial \theta_{k}}, \rho_{j}=\frac{\partial G(\theta)}{\partial \theta_{j}}$ and $\sigma_{i j}$ are elements of the covariance matrix.

For the two-parameter Weibull distribution, equation 40 reduces to

$$
\begin{aligned}
E(u(\alpha, \beta) \mid x) & =u+\frac{1}{2}\left(u_{11} \sigma_{11}+u_{22} \sigma_{22}\right)+u_{12} \sigma_{12}+u_{1}\left(\sigma_{11} \rho_{1}+\sigma_{21} \rho_{2}\right)+u_{2}\left(\sigma_{12} \rho_{1}+\sigma_{22} \rho_{2}\right) \\
& +0.5\left[L_{111}\left(u_{1} \sigma_{11}^{2}+u_{2} \sigma_{11} \sigma_{12}\right)+L_{112}\left(3 u_{1} \sigma_{11} \sigma_{12}+u_{2}\left(\sigma_{11} \sigma_{22}+2 \sigma_{12}^{2}\right)\right)\right. \\
& \left.+L_{122}\left(u_{1}\left(\sigma_{11} \sigma_{22}+2 \sigma_{12}^{2}\right)+3 u_{2} \sigma_{12} \sigma_{22}\right)+L_{222}\left(u_{1} \sigma_{12} \sigma_{22}+u_{2} \sigma_{22}^{2}\right)\right]_{\hat{\alpha}, \hat{\beta},} .
\end{aligned}
$$

Here, the $\hat{\alpha}$ and $\hat{\beta}$ are the MLEs of $\alpha$ and $\beta$, respectively.

All other quantities appearing in the above expression of $E(u(\alpha, \beta) \mid x)$ for Weibull 
distribution is given by

$$
\begin{aligned}
& \hat{\rho}_{\alpha}=\frac{a-1}{\hat{\alpha}}-b, \hat{\rho}_{\beta}=\frac{c-1}{\hat{\beta}}-d, \\
& \hat{L}_{111}=\frac{2 n}{\hat{\alpha}^{3}}-\sum_{i=1}^{n}\left(\left(\ln \left(\frac{x_{i}}{\hat{\beta}}\right)\right)^{3}\left(\frac{x_{i}}{\hat{\beta}}\right)^{\hat{\alpha}}\right), \\
& \hat{L}_{112}=\sum_{i=1}^{n} \log \left(\frac{x_{i}}{\hat{\beta}}\right)\left(\frac{x_{i}}{\hat{\beta}}\right)^{\hat{\alpha}}\left[\frac{1}{\hat{\beta}}\left(2+\hat{\alpha} \cdot \log \left(\frac{x_{i}}{\hat{\beta}}\right)\right)\right], \\
& \hat{L}_{122}=\sum_{i=1}^{n}\left(\left(\frac{x_{i}}{\hat{\beta}}\right)^{\hat{\alpha}}\left(\frac{1}{\hat{\beta}^{2}}(\hat{\alpha}+1)\left(\hat{\alpha}+\ln \left(\frac{x_{i}}{\hat{\beta}}\right)+1\right)+\hat{\alpha}\right)\right)+\frac{n^{2}}{\hat{\beta}} \\
& \hat{L}_{222}=\frac{-2 n \hat{\alpha}}{\hat{\beta}^{3}}+\frac{\hat{\alpha}(\hat{\alpha}+1)(\hat{\alpha}+2)}{\hat{\beta}^{3}} \sum_{i=1}^{n}\left(\frac{x_{i}}{\hat{\beta}}\right)^{\hat{\alpha}},
\end{aligned}
$$

and

$$
\sigma_{i j}=\left[\begin{array}{cc}
\operatorname{Var}\{\hat{\alpha}\} & \operatorname{Cov}\{\hat{\alpha}, \hat{\beta}\} \\
\operatorname{Cov}\{\hat{\alpha}, \hat{\beta}\} & \operatorname{Var}\{\hat{\beta}\}
\end{array}\right]=\frac{1}{n}\left[\begin{array}{cc}
0.6080 \alpha^{2} & 0.2570 \beta \\
0.2570 \beta & 1.1087 \frac{\beta^{2}}{\alpha^{2}}
\end{array}\right] .
$$

All constant are evaluated at $(\hat{\alpha}, \hat{\beta})$.

Then, by using Lindley's method the Bayesion estimators of the parameter $\alpha$ under SELF is obtained as

If $u(\alpha, \beta)=\alpha, u_{1}=1, u_{2}=u_{22}=u_{12}=u_{21}=u_{11}=0$, then

$$
\begin{aligned}
\hat{\alpha}_{S E L F}= & \hat{\alpha}+\left(\hat{\sigma}_{11} \hat{\rho}_{1}+\hat{\sigma}_{21} \hat{\rho}_{2}\right) \\
& +0.5\left[\hat{L}_{111} \sigma_{11}^{2}+3 \hat{L}_{112} \hat{\sigma}_{11} \hat{\sigma}_{12}+\hat{L}_{122}\left(\hat{\sigma}_{11} \hat{\sigma}_{22}+2 \hat{\sigma}_{12}^{2}\right)+\hat{L}_{222} \hat{\sigma}_{12} \hat{\sigma}_{22}\right] .
\end{aligned}
$$

So, the Bayes estimator of $\beta$ under SELF is obtained as, If $u(\alpha, \beta)=\beta, u_{2}=1, u_{22}=u_{12}=u_{21}=u_{11}=0$, then

$$
\begin{aligned}
\hat{\beta}_{S E L F}= & \hat{\beta}+\left(\hat{\sigma}_{12} \hat{\rho}_{1}+\hat{\sigma}_{22} \hat{\rho}_{2}\right) \\
& +0.5\left[\hat{L}_{111} \sigma_{11} \sigma_{12}+\hat{L}_{112}\left(\hat{\sigma}_{11} \hat{\sigma}_{22}+2 \hat{\sigma}_{12}^{2}\right)+3 \hat{L}_{122} \hat{\sigma}_{12} \hat{\sigma}_{22}+\hat{L}_{222} \hat{\sigma}_{22}^{2}\right] .
\end{aligned}
$$

Bayes estimator of $\alpha$ under the GELF is defined as

If $u(\alpha, \beta)=\alpha^{-k}, u_{1}=-k \alpha^{-(k+1)}, u_{11}=k(k+1) \alpha^{-(k+2)}, u_{2}=u_{22}=u_{12}=u_{21}=$ 0 , then

$$
\begin{aligned}
& E\left(\alpha^{-k} \mid x\right)=\hat{\alpha}^{-k}+0.5\left(\hat{u}_{11} \hat{\sigma}_{11}\right)+\hat{u}_{1}\left(\hat{\sigma}_{11} \hat{\rho}_{1}+\hat{\sigma}_{21} \hat{\rho}_{2}\right)+ \\
& 0.5\left[\hat{L}_{111} \hat{u}_{1} \hat{\sigma}_{11}^{2}+3 \hat{L}_{112} \hat{u}_{1} \hat{\sigma}_{11} \hat{\sigma}_{12}+\hat{L}_{122} \hat{u}_{1}\left(\hat{\sigma}_{11} \hat{\sigma}_{22}+2 \hat{\sigma}_{12}^{2}\right)+\hat{L}_{222} \hat{u}_{1} \hat{\sigma}_{12} \hat{\sigma}_{22}\right] .
\end{aligned}
$$

Therefore, $\hat{\alpha}_{G E L F}=E\left[\alpha^{-k} \mid x\right]^{-1 / k}$.

Bayes estimator of $\beta$ under the general entropy loss function is given by

If $u(\alpha, \beta)=\beta^{-k}, u_{2}=-k \beta^{-(k+1)}, u_{22}=k(k+1) \beta^{-(k+2)}, u_{1}=u_{11}=$ $u_{12}=u_{21}=0$, then

$$
E\left(\beta^{-k} \mid x\right)=\hat{\beta}^{-k}+0.5\left(\hat{u}_{22} \hat{\sigma}_{22}\right)+\hat{u}_{2}\left(\hat{\sigma}_{12} \hat{\rho}_{1}+\hat{\sigma}_{22} \hat{\rho}_{2}\right)+
$$




$$
0.5\left[\hat{L}_{111} \hat{u}_{2} \hat{\sigma}_{11} \hat{\sigma}_{12}+\hat{L}_{112} \hat{u}_{2}\left(\hat{\sigma}_{11} \hat{\sigma}_{22}+2 \sigma_{12}^{2}\right)+3 \hat{L}_{122} \hat{\sigma}_{12} \hat{\sigma}_{22}+\hat{L}_{222} \hat{u}_{2} \hat{\sigma}_{22}^{2}\right] \text {. }
$$

Hence, $\hat{\beta}_{G E L F}=E\left[\beta^{-k} \mid x\right]^{-1 / k}$.

Bayes estimator of $\alpha$ under the WSELF is as follows

If $u(\alpha, \beta)=\alpha^{-1}, u_{1}=-\alpha^{-2}, u_{11}=2 \alpha^{-3}, u_{2}=u_{22}=u_{12}=0$, then

$$
\begin{aligned}
& E\left(\alpha^{-1} \mid x\right)=\hat{\alpha}^{-1}+0.5\left(\hat{u}_{11} \hat{\sigma}_{11}\right)+\hat{u}_{1}\left(\hat{\sigma}_{11} \hat{\rho}_{1}+\hat{\sigma}_{21} \hat{\rho}_{2}\right)+ \\
& 0.5\left[\hat{L}_{111} \hat{u}_{1} \hat{\sigma}_{11}^{2}+3 \hat{L}_{112} \hat{u}_{1}\left(\hat{\sigma}_{11} \hat{\sigma}_{12}+\hat{L}_{122} \hat{u}_{1}\left(\hat{\sigma}_{11} \hat{\sigma}_{22}+2 \hat{\sigma}_{12}^{2}\right)+\hat{L}_{222} \hat{u}_{1} \hat{\sigma}_{12} \hat{\sigma}_{22}\right] .\right.
\end{aligned}
$$

So, $\hat{\alpha}_{W S E L F}=\left[E\left(\alpha^{-1} \mid x\right)\right]^{-1}$.

The Bayes estimator of $\beta$ under the WSELF is given in following form

If $u(\alpha, \beta)=\beta^{-1}, u_{2}=-\beta^{-2}, u_{22}=2 \beta^{-3}, u_{1}=u_{11}=u_{12}=0$, then

$$
\begin{aligned}
& E\left(\beta^{-1} \mid x\right)=\hat{\beta}^{-1}+0.5\left(\hat{u}_{22} \hat{\sigma}_{22}\right)+\hat{u}_{2}\left(\hat{\sigma}_{12} \hat{\rho}_{1}+\hat{\sigma}_{22} \hat{\rho}_{2}\right)+ \\
& 0.5\left[\hat{L}_{111} \hat{u}_{2} \hat{\sigma}_{11} \hat{\sigma}_{12}+\hat{L}_{112} \hat{u}_{2}\left(\hat{\sigma}_{11} \hat{\sigma}_{22}+2 \hat{\sigma}_{12}^{2}\right)+3 \hat{L}_{122} \hat{\sigma}_{12} \hat{\sigma}_{22}+\hat{L}_{222} \hat{u}_{2} \hat{\sigma}_{22}^{2}\right] .
\end{aligned}
$$

So, the Bayes estimator of $\beta$ is $\hat{\beta}_{W S E L F}=\left[E\left(\beta^{-1} \mid x\right)\right]^{-1}$.

Finally, the Bayes estimator of $\alpha$ under PLF is

If $u(\alpha, \beta)=\alpha^{2}, u_{1}=2 \alpha, u_{11}=2, u_{2}=u_{22}=u_{12}=0$, then

$$
\begin{aligned}
& E\left(\alpha^{2} \mid x\right)=\hat{\alpha}^{2}+0.5\left(\hat{u}_{11} \hat{\sigma}_{11}\right)+\hat{u}_{1}\left(\hat{\sigma}_{11} \hat{\rho}_{1}+\hat{\sigma}_{21} \hat{\rho}_{2}\right)+ \\
& 0.5\left[\hat{L}_{111} \hat{u}_{1} \hat{\sigma}_{11}^{2}+3 \hat{L}_{112} \hat{u}_{1} \hat{\sigma}_{11} \hat{\sigma}_{12}+\hat{L}_{122} \hat{u}_{1}\left(\hat{\sigma}_{11} \hat{\sigma}_{22}+2 \hat{\sigma}_{12}^{2}\right)+\hat{L}_{222} \hat{u}_{1} \hat{\sigma}_{12} \hat{\sigma}_{22}\right] .
\end{aligned}
$$

Hence,the Bayes estimator of $\alpha$ is as follows $\hat{\alpha}_{P L F}=\sqrt{E\left(\alpha^{2} \mid x\right)}$.

Bayes estimator of $\beta$ under PLF is given by If $u(\alpha, \beta)=\beta^{2}, u_{2}=2 \beta, u_{22}=2, u_{1}=u_{11}=u_{12}=0$, then

$$
\begin{aligned}
& E(\beta \mid x)=\hat{\beta}+0.5\left(\hat{u}_{22} \hat{\sigma}_{22}\right)+\hat{u}_{2}\left(\hat{\sigma}_{12} \hat{\rho}_{1}+\hat{\sigma}_{22} \hat{\rho}_{2}\right)+ \\
& 0.5\left[\hat{L}_{111} \hat{u}_{2} \hat{\sigma}_{11} \hat{\sigma}_{12}+\hat{L}_{112} \hat{u}_{2}\left(\hat{\sigma}_{11} \hat{\sigma}_{22}+2 \hat{\sigma}_{12}^{2}\right)+3 \hat{L}_{122} \hat{\sigma}_{12} \hat{\sigma}_{22}+\hat{L}_{222} \hat{u}_{2} \hat{\sigma}_{22}^{2}\right] .
\end{aligned}
$$

So, $\hat{\beta}_{P L F}=\sqrt{E\left(\beta^{2} \mid x\right)}$.

4.2 Tierney Kadane's Procedure. Lindley's procedure seems to be become more and more complex in p- parameter case $(p>2)$. Therefore, in multi-parameter case, Tierney Kadane's (T-K) procedure is used as an alternative to Lindley's procedure [47, 48].

According to this procedure, posterior expectation for multi-parameter case can be approximated by:

$$
E(u(\theta) \mid x)=\sqrt{\frac{\left|\Sigma^{*}\right|}{|\Sigma|}} \exp \left[n\left(L_{1}^{*}\left(\hat{\theta}^{*}\right)-L_{1}(\hat{\theta})\right)\right] .
$$


Here, $\hat{\theta}^{*}$ and $\hat{\theta}$ maximize $L_{1}^{*}$ and $L_{1}$,respectively,

$$
L_{1}=\frac{[L(\theta)+\log (v(\theta))]}{n}, L_{1}^{*}=L_{1}+\frac{[\log (u(\theta))]}{n},
$$

where

$v(\theta)=$ joint prior distribution of $\theta$,

$L(\theta)=$ Log-likelihood function of $\theta$,

$u(\theta)=$ loss function of $\theta$.

In equation $42, \sum^{*}$ and $\sum$ are elements of the negative of the inverse of the matrices of the second derivatives of $L_{1}^{*}$ and $L_{1}$ at the point $\hat{\theta}^{*}$ and $\theta$, respectively.

For the two parameter case, $\theta=(\alpha, \beta)$, equation 42 becomes:

$$
E(u(\alpha, \beta) \mid x)=\sqrt{\frac{\left|\sum^{*}\right|}{\left|\sum\right|}} \exp \left[n\left(L_{1}^{*}\left(\hat{\alpha}^{*}, \hat{\beta}^{*}\right)-L_{1}(\hat{\alpha}, \hat{\beta})\right)\right] .
$$

Here, $(\hat{\beta}, \hat{\alpha})$ and $\left(\hat{\beta}^{*}, \hat{\alpha}^{*}\right)$ maximize $L_{1}(\alpha, \beta)$ and $L_{1}^{*}(\alpha, \beta)$, respectively. $\sum$ and $\sum^{*}$ are given below:

$$
\sum^{*}=\left[\begin{array}{rr}
-\frac{\partial^{2} L_{1}^{*}}{\partial \alpha^{2}} & -\frac{\partial^{2} L_{1}^{*}}{\partial \alpha \partial \beta} \\
-\frac{\partial^{2} L_{1}^{*}}{\partial \alpha \partial \beta} & -\frac{\partial^{2} L_{1}^{*}}{\partial \beta}
\end{array}\right]_{\left(\hat{\alpha}^{*}, \hat{\beta}^{*}\right)}^{-1} \text { and } \sum=\left[\begin{array}{ll}
-\frac{\partial^{2} L_{1}}{\partial \alpha^{2}} & -\frac{\partial^{2} L_{1}}{\partial \partial \beta} \\
-\frac{\partial^{2} L_{1}}{\partial \alpha \partial \alpha} & -\frac{\partial^{2} L_{1}}{\partial \beta^{2}}
\end{array}\right]_{(\hat{\alpha}, \hat{\beta})}^{-1}
$$

All other quantities appearing in the above expression of $E(u(\alpha, \beta \mid x))$ for Weibull distribution can be obtained as

$L_{1}(\alpha, \beta)=\frac{1}{n}\left[n \ln \alpha-n \alpha \ln \beta+(\alpha-1) \sum_{i=1}^{n} x_{i}-\sum_{i=1}^{n}\left(\frac{x_{i}}{\beta}\right)^{\alpha}+(\alpha-1) \ln \alpha+(c-1) \ln \beta-(b \alpha+d \beta)\right]$.

Thus the Bayes estimator of $\alpha$ under SELF is given in the following form:

If $u(\alpha, \beta)=\alpha$ and $L_{1}^{*}=\frac{1}{n} \log \alpha+L_{1}(\alpha, \beta)$, then

$$
\hat{\alpha}_{S E L F}=\left[\sqrt{\frac{\left|\sum^{*}\right|}{\left|\sum\right|}} \exp \left[n\left(\frac{1}{n} \log \hat{\alpha}+L_{1}\left(\hat{\alpha}^{*}, \hat{\beta}^{*}\right)-L_{1}(\hat{\alpha}, \hat{\beta})\right)\right]\right] .
$$

Also, the Bayes estimator of $\beta$ under SELF using this procedure is defined as: If $u(\alpha, \beta)=\beta$ and $L_{1}^{*}=\frac{1}{n} \log \beta+L_{1}(\alpha, \beta)$, then

$$
\hat{\beta}_{S E L F}=\left[\sqrt{\frac{\left|\sum^{*}\right|}{\left|\sum\right|}} \exp \left[n\left(\frac{1}{n} \log \hat{\beta}+L_{1}\left(\hat{\alpha}^{*}, \hat{\beta}^{*}\right)-L_{1}(\hat{\alpha}, \hat{\beta})\right)\right]\right] .
$$

Bayes estimator of $\alpha$ under GELF is given by:

If $u(\alpha, \beta)=\alpha^{-k}$ and $L_{1}^{*}=\frac{1}{n} \log \left(\alpha^{-k}\right)+L_{1}(\alpha, \beta)$, then

$$
\hat{\alpha}_{G E L F}=\left[\sqrt{\frac{\left|\sum^{*}\right|}{\left|\sum\right|}} \exp \left[n\left(\frac{1}{n} \log \left(\hat{\alpha}^{-k}\right)+L_{1}^{*}\left(\hat{\alpha}^{*}, \hat{\beta}^{*}\right)-L_{1}(\hat{\alpha}, \hat{\beta})\right)\right]\right]^{-1 / k} .
$$


Bayes estimator of $\beta$ under GELF is given by:

If $u(\alpha, \beta)=\beta^{-k}$ and $L_{1}^{*}=\frac{1}{n} \log \left(\beta^{-k}\right)+L_{1}(\alpha, \beta)$, then

$$
\hat{\beta}_{G E L F}=\left[\sqrt{\frac{\left|\sum^{*}\right|}{\left|\sum\right|}} \exp \left[n\left(\frac{1}{n} \log \left(\hat{\beta}^{-k}\right)+L_{1}^{*}\left(\hat{\alpha}^{*}, \hat{\beta}^{*}\right)-L_{1}(\hat{\alpha}, \hat{\beta})\right)\right]\right]^{-1 / k} .
$$

Bayes estimator of $\alpha$ under WSELF is as follows:

If $u(\alpha, \beta)=\alpha^{-1}$ and $L_{1}^{*}=\frac{1}{n} \log \left(\alpha^{-1}\right)+L_{1}(\alpha, \beta)$, then

$$
\hat{\alpha}_{W S E L F}=\left[\sqrt{\frac{\left|\sum^{*}\right|}{\left|\sum\right|}} \exp \left[n\left(\frac{1}{n} \log \left(\hat{\alpha}^{-1}\right)+L_{1}^{*}\left(\hat{\alpha}^{*}, \hat{\beta}^{*}\right)-L_{1}(\hat{\alpha}, \hat{\beta})\right)\right]\right]^{-1} .
$$

Bayes estimator of $\beta$ under WSELF is as follows:

If $u(\alpha, \beta)=\beta^{-1}$ and $L_{1}^{*}=\frac{1}{n} \log (\beta)^{-1}+L_{1}(\alpha, \beta)$, then

$$
\left.\hat{\beta}_{W S E L F}=\left[\sqrt{\frac{\left|\sum *\right|}{\left|\sum\right|}} \exp \left[n\left(\frac{1}{n} \log \hat{\left(\beta^{-1}\right.}\right)+L_{1}^{*}\left(\hat{\alpha}^{*}, \hat{\beta}^{*}\right)-L_{1}(\hat{\alpha}, \hat{\beta})\right)\right]\right]^{-1} .
$$

Bayes estimator of $\alpha$ under PLF is

If $u(\alpha, \beta)=\alpha^{2}$ and $L_{1}^{*}=\frac{1}{n} \log (\alpha)^{2}+L_{1}(\alpha, \beta)$, then

$$
\hat{\alpha}_{P L F}=\sqrt{\sqrt{\frac{\left|\sum^{*}\right|}{\left|\sum\right|}} \exp \left[n\left(\frac{1}{n} \log \left(\hat{\alpha}^{2}\right)+L_{1}^{*}\left(\hat{\alpha}^{*}, \hat{\beta}^{*}\right)-L_{1}(\hat{\alpha}, \hat{\beta})\right)\right] .}
$$

Bayes estimator of $\beta$ under PLF is

If $u(\alpha, \beta)=\beta^{2}$ and $L_{1}^{*}=\frac{1}{n} \log (\beta)^{2}+L_{1}(\alpha, \beta)$, then

$$
\hat{\beta}_{P L F}=\sqrt{\sqrt{\frac{\left|\sum^{*}\right|}{\left|\sum\right|}} \exp \left[n\left(\frac{1}{n} \log \left(\hat{\beta}^{2}\right)+L_{1}^{*}\left(\hat{\alpha}^{*}, \hat{\beta}^{*}\right)-L_{1}(\hat{\alpha}, \hat{\beta})\right)\right]} .
$$

\section{Simulation Study}

In this section, an extensive Monte Carlo simulation study was carried out to compare the performances of the Bayesian and classical estimators with respect to the biases and mean squared errors (MSEs) for different sample sizes and parameter values. All The computations were performed in Matlab R. 2013. over 10.000 replications for different cases. We consider the sample sizes $n=10(10) 100$, the shape parameter values $\alpha=0.5,1.5$ and the scale parameter $\beta$ was taken to be 1 throughout the study. The bias and MSE values of the classical estimators are given in Table 1.

For Bayesian estimators, we know that the Gamma prior provides flexible approach in both informative and non-informative cases [48. In case of the noninformative prior (NP), we chose hyper-parameter values as $a=b=c=d=0$. In case of the GP, we chose hyper-parameter values as $a=0.4,1,1.5,3, b=0.2,1$, $c=0.4,1,1.5,3$ and $d=0.2,1$. In both cases i.e. informative and non-informative, 
we considered as $k= \pm 1.5$ for GELF. Because of the large number of tables and results, only results for $a=c=0.4, b=d=0.2$ and $k=1.5$ were reported. Moreover, Lindley's and T-K methods were used to obtain the Bayes estimator of the unknown parameters. The results of simulation for these approximation methods were summarized in Table 2-3.

Table 1. The simulated, means and MSEs values for the classical different parameter estimators of $\alpha$ and $\beta$

\begin{tabular}{|c|c|c|c|c|c|c|}
\hline & & & \multicolumn{2}{|c|}{$\hat{\alpha}$} & \multicolumn{2}{|c|}{$p$} \\
\hline $\mathrm{n}$ & $\alpha$ & Estimator & Mean & MSE & Mean & MSE \\
\hline \multirow{9}{*}{20} & \multirow{9}{*}{0.5} & MLE & 0.5382 & 0.0121 & 1.0704 & 0.2607 \\
\hline & & LME & 0.5290 & 0.0152 & 0.9837 & 0.2598 \\
\hline & & TLME & 0.5079 & 0.0138 & 0.9579 & 0.2395 \\
\hline & & MMLE-II & 0.5330 & 0.0123 & 1.0687 & 0.2610 \\
\hline & & MMLE-I & 0.4739 & 0.0220 & 0.9874 & 0.2616 \\
\hline & & ME & 0.6511 & 0.0403 & 1.3510 & 0.5322 \\
\hline & & LSE & 0.5008 & 0.0147 & 1.1271 & 0.3244 \\
\hline & & WLSE & 0.5052 & 0.0131 & 1.1114 & 0.2973 \\
\hline & & $\mathrm{PE}$ & 0.4887 & 0.0336 & 1.1229 & 0.2395 \\
\hline \multirow{9}{*}{30} & \multirow{9}{*}{0.5} & MLE & 0.5243 & 0.0068 & 1.0544 & 0.1694 \\
\hline & & LME & 0.5210 & 0.0101 & 0.9960 & 0.1752 \\
\hline & & TLME & 0.5039 & 0.0081 & 0.9807 & 0.1622 \\
\hline & & MMLE-II & 0.5226 & 0.0069 & 1.0541 & 0.1700 \\
\hline & & MMLE-I & 0.4702 & 0.0172 & 0.9816 & 0.1779 \\
\hline & & $\mathrm{ME}$ & 0.6151 & 0.0263 & 1.2863 & 0.3425 \\
\hline & & LSE & 0.4984 & 0.0087 & 1.0932 & 0.2037 \\
\hline & & WLSE & 0.5034 & 0.0076 & 1.0795 & 0.1878 \\
\hline & & $\mathrm{PE}$ & 0.4800 & 0.0276 & 1.0577 & 0.2268 \\
\hline \multirow{9}{*}{50} & \multirow{9}{*}{0.5} & MLE & 0.5147 & 0.0037 & 1.0276 & 0.0951 \\
\hline & & LME & 0.5125 & 0.0059 & 0.9891 & 0.1019 \\
\hline & & TLME & 0.5032 & 0.0045 & 0.9829 & 0.0945 \\
\hline & & MMLE-II & 0.5139 & 0.0038 & 1.0280 & 0.0959 \\
\hline & & MMLE-I & 0.4689 & 0.0139 & 0.9654 & 0.1154 \\
\hline & & $\mathrm{ME}$ & 0.5782 & 0.0149 & 1.1955 & 0.1874 \\
\hline & & LSE & 0.5000 & 0.0048 & 1.0490 & 0.1094 \\
\hline & & WLSE & 0.5039 & 0.0041 & 1.0401 & 0.1014 \\
\hline & & $\mathrm{PE}$ & 0.4664 & 0.0210 & 0.9622 & 0.3263 \\
\hline
\end{tabular}


Table 1. Continued

\begin{tabular}{|c|c|c|c|c|c|c|}
\hline \multirow[b]{2}{*}{$\mathrm{n}$} & \multirow[b]{2}{*}{$\alpha$} & \multirow[b]{2}{*}{ Estimator } & \multicolumn{2}{|c|}{$\hat{\alpha}$} & \multicolumn{2}{|c|}{$\hat{\beta}$} \\
\hline & & & Mean & MSE & Mean & MSE \\
\hline \multirow{9}{*}{100} & \multirow{9}{*}{0.5} & MLE & 0.5068 & 0.0016 & 1.0112 & 0.0458 \\
\hline & & LME & 0.5068 & 0.0029 & 0.9931 & 0.0506 \\
\hline & & TLME & 0.5012 & 0.0022 & 0.9894 & 0.0472 \\
\hline & & MMLE-II & 0.5063 & 0.0017 & 1.0111 & 0.0461 \\
\hline & & MMLE-I & 0.4697 & 0.0106 & 0.9586 & 0.0661 \\
\hline & & $\mathrm{ME}$ & 0.5487 & 0.0076 & 1.1264 & 0.0959 \\
\hline & & LSE & 0.4994 & 0.0023 & 1.0223 & 0.0516 \\
\hline & & WLSE & 0.5020 & 0.0019 & 1.0170 & 0.0481 \\
\hline & & $\mathrm{PE}$ & 0.4653 & 0.0142 & 0.9208 & 0.1755 \\
\hline \multirow{9}{*}{20} & \multirow{9}{*}{1.5} & MLE & 1.6090 & 0.1041 & 1.0010 & 0.0245 \\
\hline & & LME & 1.5292 & 0.0878 & 0.9943 & 0.0247 \\
\hline & & TLME & 1.5333 & 0.1304 & 0.9968 & 0.0270 \\
\hline & & MMLE-II & 1.5989 & 0.1055 & 1.0008 & 0.0246 \\
\hline & & MMLE-I & 1.4225 & 0.1959 & 0.9712 & 0.0281 \\
\hline & & $\mathrm{ME}$ & 1.6194 & 0.1051 & 1.0016 & 0.0245 \\
\hline & & LSE & 1.4949 & 0.1262 & 1.0165 & 0.0276 \\
\hline & & WLSE & 1.5087 & 0.1116 & 1.0129 & 0.0262 \\
\hline & & $\mathrm{PE}$ & 1.4362 & 0.0968 & 1.0172 & 0.0734 \\
\hline \multirow{9}{*}{30} & \multirow{9}{*}{1.5} & MLE & 1.5740 & 0.0625 & 0.9999 & 0.0166 \\
\hline & & LME & 1.5225 & 0.0563 & 0.9956 & 0.0167 \\
\hline & & TLME & 1.5218 & 0.0793 & 0.9977 & 0.0179 \\
\hline & & MMLE-II & 1.5689 & 0.0634 & 0.9998 & 0.0167 \\
\hline & & MMLE-I & 1.4112 & 0.1556 & 0.9732 & 0.0202 \\
\hline & & $\mathrm{ME}$ & 1.5826 & 0.0651 & 1.0003 & 0.0167 \\
\hline & & LSE & 1.4981 & 0.0787 & 1.0104 & 0.0183 \\
\hline & & WLSE & 1.5125 & 0.0683 & 1.0071 & 0.0174 \\
\hline & & $\mathrm{PE}$ & 1.4471 & 0.0667 & 1.0107 & 0.0021 \\
\hline \multirow{9}{*}{50} & \multirow{9}{*}{1.5} & MLE & 1.5438 & 0.0331 & 0.9995 & 0.0099 \\
\hline & & LME & 1.5137 & 0.0319 & 0.9969 & 0.0100 \\
\hline & & TLME & 1.5133 & 0.0445 & 0.9982 & 0.0108 \\
\hline & & MMLE-II & 1.5413 & 0.0344 & 0.9994 & 0.0100 \\
\hline & & MMLE-I & 1.4103 & 0.1258 & 0.9763 & 0.0133 \\
\hline & & $\mathrm{ME}$ & 1.5496 & 0.0353 & 0.9997 & 0.0100 \\
\hline & & LSE & 1.4989 & 0.0450 & 1.0058 & 0.0110 \\
\hline & & WLSE & 1.5107 & 0.0381 & 1.0034 & 0.0104 \\
\hline & & $\mathrm{PE}$ & 1.4549 & 0.0398 & 1.0057 & 0.1723 \\
\hline \multirow{9}{*}{100} & \multirow{9}{*}{1.5} & MLE & 1.5213 & 0.0151 & 1.0000 & 0.0050 \\
\hline & & LME & 1.5065 & 0.0152 & 0.9988 & 0.0050 \\
\hline & & TLME & 1.5067 & 0.0206 & 0.9993 & 0.0054 \\
\hline & & MMLE-II & 1.5199 & 0.0158 & 1.0000 & 0.0050 \\
\hline & & MMLE-I & 1.4063 & 0.0976 & 0.9790 & 0.0081 \\
\hline & & $\mathrm{ME}$ & 1.5244 & 0.0165 & 1.0001 & 0.0050 \\
\hline & & LSE & 1.4998 & 0.0213 & 1.003 & 0.0055 \\
\hline & & WLSE & 1.5075 & 0.0175 & 1.0017 & 0.0052 \\
\hline & & $\mathrm{PE}$ & 1.4658 & 0.0205 & 1.0025 & 0.0735 \\
\hline
\end{tabular}


Table 2.The simulated, means and MSEs values under different loss function for the Lindley approximation of $\alpha$ and $\beta$

\begin{tabular}{|c|c|c|c|c|c|c|c|c|c|c|}
\hline & & & \multicolumn{8}{|c|}{ Lindley's approximation } \\
\hline & & & \multicolumn{4}{|c|}{$\hat{\alpha}$} & \multicolumn{4}{|c|}{$\hat{\beta}$} \\
\hline & & & \multicolumn{2}{|c|}{ NP } & \multicolumn{2}{|c|}{ GP } & \multicolumn{2}{|c|}{ NP } & \multicolumn{2}{|c|}{ GP } \\
\hline $\mathrm{n}$ & $\alpha$ & $\overline{\mathrm{LF}}$ & Mean & MSE & Mean & MSE & Mean & MSE & Mean & MSE \\
\hline \multirow{4}{*}{20} & \multirow{4}{*}{0.5} & SELF & 0.5178 & 0.0102 & 0.5240 & 0.0100 & 1.1895 & 0.3391 & $\overline{1.2313}$ & 0.3487 \\
\hline & & GELF & 0.5008 & 0.0092 & 0.5060 & 0.0089 & 0.9441 & 0.2152 & 0.9746 & 0.2060 \\
\hline & & WSELF & 0.5038 & 0.0094 & 0.5092 & 0.0090 & 0.9825 & 0.2258 & 1.0182 & 0.2177 \\
\hline & & PLF & 0.5258 & 0.0108 & 0.5321 & 0.0108 & 1.2758 & 0.4192 & 1.3101 & 0.4347 \\
\hline \multirow{4}{*}{30} & \multirow{4}{*}{0.5} & SELF & 0.5110 & 0.0061 & 0.5154 & 0.0062 & 1.1349 & 0.2053 & 1.1635 & 0.2104 \\
\hline & & GELF & 0.4993 & 0.0057 & 0.5032 & 0.0057 & 0.9640 & 0.1468 & 0.9868 & 0.1429 \\
\hline & & WSELF & 0.5015 & 0.0057 & 0.5054 & 0.0058 & 0.9930 & 0.1524 & 1.0186 & 0.1494 \\
\hline & & PLF & 0.5163 & 0.0063 & 0.5207 & 0.0065 & 1.1976 & 0.2441 & 1.2220 & 0.2518 \\
\hline \multirow{4}{*}{50} & \multirow{4}{*}{0.5} & SELF & 0.5068 & 0.0034 & 0.5097 & 0.0035 & 1.0759 & 0.1071 & 1.0974 & 0.1128 \\
\hline & & GELF & 0.4996 & 0.0033 & 0.5023 & 0.0033 & 0.9709 & 0.0875 & 0.9897 & 0.0880 \\
\hline & & WSELF & 0.5009 & 0.0033 & 0.5037 & 0.0033 & 0.9899 & 0.0894 & 1.0099 & 0.0906 \\
\hline & & PLF & 0.5099 & 0.0035 & 0.5129 & 0.0036 & 1.1157 & 0.1209 & 1.1356 & 0.1284 \\
\hline \multirow{4}{*}{100} & \multirow{4}{*}{0.5} & SELF & 0.5029 & 0.0016 & 0.5031 & 0.0016 & 1.0354 & 0.0487 & 1.0418 & 0.0507 \\
\hline & & GELF & 0.4992 & 0.0015 & 0.4994 & 0.0016 & 0.9816 & 0.0441 & 0.9876 & 0.0453 \\
\hline & & WSELF & 0.4999 & 0.0015 & 0.5002 & 0.0016 & 0.9918 & 0.0445 & 0.9979 & 0.0459 \\
\hline & & PLF & 0.5045 & 0.0016 & 0.5047 & 0.0016 & 1.0564 & 0.0521 & 1.0628 & 0.0545 \\
\hline \multirow{4}{*}{20} & \multirow{4}{*}{1.5} & SELF & 1.5480 & 0.0877 & 1.5585 & 0.0894 & 1.0161 & 0.0250 & 1.0177 & 0.0250 \\
\hline & & GELF & 1.4974 & 0.0799 & 1.5069 & 0.0807 & 0.9874 & 0.0245 & 0.9889 & 0.0243 \\
\hline & & WSELF & 1.5061 & 0.0809 & 1.5158 & 0.0818 & 0.9930 & 0.0245 & 0.9946 & 0.0243 \\
\hline & & PLF & 1.5720 & 0.0932 & 1.5828 & 0.0955 & 1.0274 & 0.0258 & 1.0287 & 0.0258 \\
\hline \multirow{4}{*}{30} & \multirow{4}{*}{1.5} & SELF & 1.5340 & 0.0553 & 1.5387 & 0.0548 & 1.0102 & 0.0169 & 1.0122 & 0.0163 \\
\hline & & GELF & 1.4989 & 0.0517 & 1.5031 & 0.0509 & 0.9906 & 0.0166 & 0.9927 & 0.0160 \\
\hline & & WSELF & 1.5053 & 0.0521 & 1.5096 & 0.0514 & 0.9945 & 0.0166 & 0.9965 & 0.0160 \\
\hline & & PLF & 1.5498 & 0.0577 & 1.5545 & 0.0574 & 1.0179 & 0.0172 & 1.0199 & 0.0166 \\
\hline \multirow{4}{*}{50} & \multirow{4}{*}{1.5} & SELF & 1.5202 & 0.0306 & 1.5229 & 0.0300 & 1.0057 & 0.0100 & 1.0090 & 0.0100 \\
\hline & & GELF & 1.4984 & 0.0294 & 1.5010 & 0.0287 & 0.9938 & 0.0099 & 0.9970 & 0.0098 \\
\hline & & WSELF & 1.5025 & 0.0295 & 1.5051 & 0.0288 & 0.9961 & 0.0099 & 0.9994 & 0.0098 \\
\hline & & PLF & 1.5295 & 0.0315 & 1.5322 & 0.0309 & 1.0104 & 0.0101 & 1.0137 & 0.0101 \\
\hline \multirow{4}{*}{100} & \multirow{4}{*}{1.5} & SELF & 1.5096 & 0.0145 & 1.5119 & 0.0150 & 1.0032 & 0.0050 & 1.0037 & $\overline{0.0049}$ \\
\hline & & GELF & 1.4985 & 0.0142 & 1.5008 & 0.0146 & 0.9971 & 0.0050 & 0.9976 & 0.0049 \\
\hline & & WSELF & 1.5006 & 0.0142 & 1.5029 & 0.0147 & 0.9983 & 0.0050 & 0.9988 & 0.0049 \\
\hline & & PLF & 1.5142 & 0.0147 & 1.5166 & 0.0152 & 1.0056 & 0.0051 & 1.0061 & 0.0049 \\
\hline
\end{tabular}

In all cases, the biases and MSEs of the estimators decrease as the sample size $\mathrm{n}$ increases. It indicates that all the estimators are asymptotically unbiased and 
Table 3.The simulated, means and MSEs values under different loss function for Tierney Kadaneâ $€^{\mathrm{TM}} \mathrm{S}$ approximation parameter estimators of $\alpha$ and $\beta$

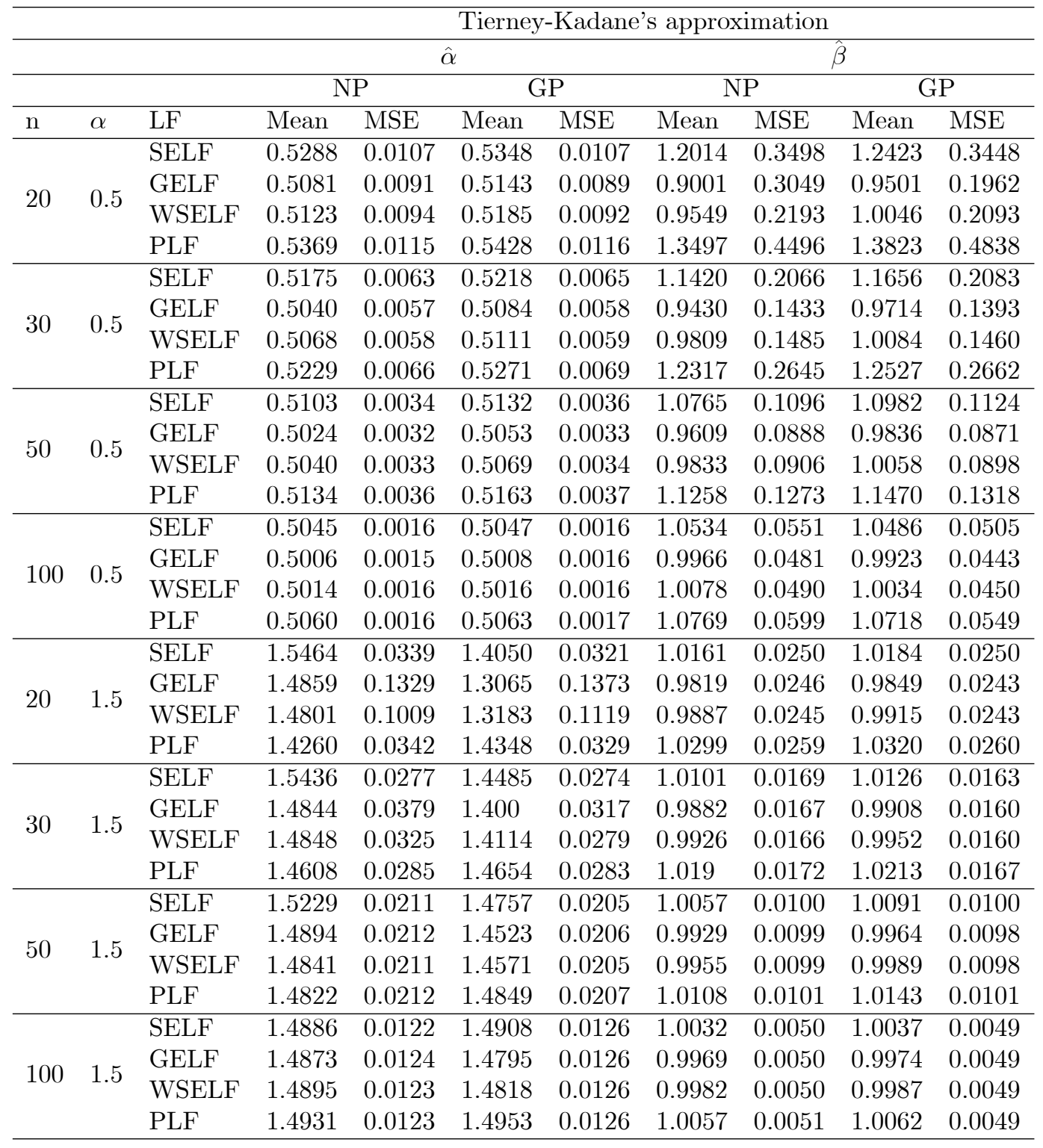


consistent for the parameters $\alpha$ and $\beta$. When the classical methods are compared with each other, for the shape parameter $\alpha$, as far as bias is concerned, LSE, WLSE and TLME work the best for all sample sizes. With respect to the MSEs, for $\alpha<1$, MMLE-II performs better than the other estimators for small sample sizes $(n<20)$ and otherwise MLE outperforms the rest. For $\alpha \geq 1$, LME works the best for small sample sizes $(n \leq 20)$. For large sample sizes $(n \geq 50)$, MLE and MMLE-II both works very well.

Similarly, if we compare the classical estimators for $\beta$, comparing the biases, for $\alpha<1$, it is observed that LME and TLME work the best for particularly small sample sizes and for large sample sizes $(n>50)$, the performances of the LME and TLME are close to that of the MLE and MMLE-II. When $\alpha \geq 1$, LME and TLME work the better than the other estimators for small sample sizes $(n \leq 20)$ and otherwise MLE and MMLE-II outperform the rest.

Then, if we compare the performance of Bayes estimators obtained by Lindley's method, it is clear that as far as MSE and bias are concerned, Bayes estimators under GELF and WSELF work the best in all cases. Similarly, comparing the performance of Bayes estimators obtained by Tierney Kadane's approximation, it is observed that, if $\alpha \leq 1$, Bayes estimators obtained under GELF works the best in all cases for estimating $\alpha$ parameter, followed by Bayes estimation under the WSELF. When $\alpha>1$, for estimating parameter, Bayesian estimations under SELF and PLF work very well.

For estimating $\beta$ parameter, Bayes estimation under WSELF performs better than the other estimators for small sample sizes $(n \leq 20)$ and otherwise Bayesian estimations under WSELF and GELF give the same result.

When we compare the Bayesian and classical methods for estimating the $\alpha$ and $\beta$ parameters, it is clear that as far as bias and MSE are concerned; Bayesian methods outperform the classical methods. Furthermore, Lindley's method works well than the Tierney-Kadane's method in the most of the cases. Also, the GP gives better estimators than the NP for all loss functions.

\section{Application}

In this section, an actual data set is used to illustrate the estimation procedure developed in section 3-4. The data set measured from Sivas, Turkey during 2017 was used. There were 6011 observations recorded. The data was taken from the Turkish State Meteorological Service. All measurements were made at the heights of $10 \mathrm{~m}$ in hourly basis.

In this paper, the performance of the Weibull distribution (WD) was compared with the Gamma distribution (GD), log- normal distribution (LND) and inverse Gauss distribution (IGD). These distributions for wind speed data were analyzed seasonally and annually. To determine the distribution providing better fit to wind speed data, we computed the root mean square error (RMSE), the coefficient of 
determination $\left(R^{2}\right)$ and Akaike information criteria(AIC) values for each distribution, as shown in Table 4. The formulas for model selection criteria were given in Table 5. In addition to these statistical criteria, the cumulative density function of the WD, GD, IGD and LND were presented in Figure 1 for seasonal and annual wind speed data.

Table 4. RMSE, $R^{2}$ and AIC values for distributions

\begin{tabular}{lllllll}
\hline $\mathrm{n}$ & & Criteria & WD & IGD & LN & GD \\
\hline \multirow{3}{*}{6011} & \multirow{2}{*}{ Annual } & RMSE & 0.0215 & 0.0746 & 0.0502 & 0.0301 \\
& & R2 & 0.9946 & 0.9214 & 0.9664 & 0.989 \\
& & AIC & 2.5736 & 2.7704 & 2.7488 & 2.6058 \\
\hline \multirow{3}{*}{1560} & \multirow{2}{*}{ Winter } & RMSE & 0.0215 & 0.0821 & 0.0571 & 0.0353 \\
& & R2 & 0.9945 & 0.9053 & 0.9564 & 0.9847 \\
& & AIC & 6.6889 & 7.2235 & 6.9984 & 6.7801 \\
\hline \multirow{3}{*}{1710} & \multirow{2}{*}{ Spring } & RMSE & 0.025 & 0.0627 & 0.0419 & 0.0261 \\
& & R2 & 0.9926 & 0.9453 & 0.977 & 0.9918 \\
& & AIC & 7.2749 & 7.7628 & 8.3423 & 7.3414 \\
\hline \multirow{3}{*}{1429} & \multirow{2}{*}{ Summer } & RMSE & 0.0236 & 0.0796 & 0.0538 & 0.0338 \\
& & R2 & 0.9936 & 0.9084 & 0.9606 & 0.986 \\
& & AIC & 6.1904 & 6.7319 & 6.8202 & 6.298 \\
\hline \multirow{3}{*}{1312} & \multirow{2}{*}{ Autumn } & RMSE & 0.0231 & 0.0721 & 0.0482 & 0.0287 \\
& & R2 & 0.9936 & 0.9276 & 0.9693 & 0.99 \\
& & AIC & 5.5252 & 5.8949 & 5.6003 & 5.5729 \\
\hline & & & & & & \\
\hline
\end{tabular}

Table 5. The formulas of criteria for model evaluation

\begin{tabular}{ll}
\hline Criteria & Formulas \\
\hline RMSE & $2 k-2 \ln \alpha$ \\
$R^{2}$ & $1-\left(\sum_{i=1}^{n} \hat{F}\left(X_{(i)}\right)-\frac{i}{n+1}\right)^{2} /\left(\sum_{i=1}^{n} \hat{F}\left(X_{i}\right)-\overline{\hat{F}}\left(X_{i}\right)\right)^{2}$ \\
AIC & {$\left[\sum_{i=1}^{n}\left(\hat{F}\left(X_{(i)}\right)-\frac{i}{n+1}\right)^{2} / n\right]^{1 / 2}$} \\
\hline
\end{tabular}

According to Table 4, Weibull distribution has the smallest RMSE, AIC values and the highest $R^{2}$ values. In Table $5, k$ is the number of the unknown parameters, In $L$ is the value of log-likelihood function for each distribution, $\hat{F}$ is the estimated cumulative density function, $X_{i}$ is $i-t h$ order statistics, $n$ is sample size and $\overline{\hat{F}}=\sum_{i=1}^{n} \hat{F}_{i} / n$. 

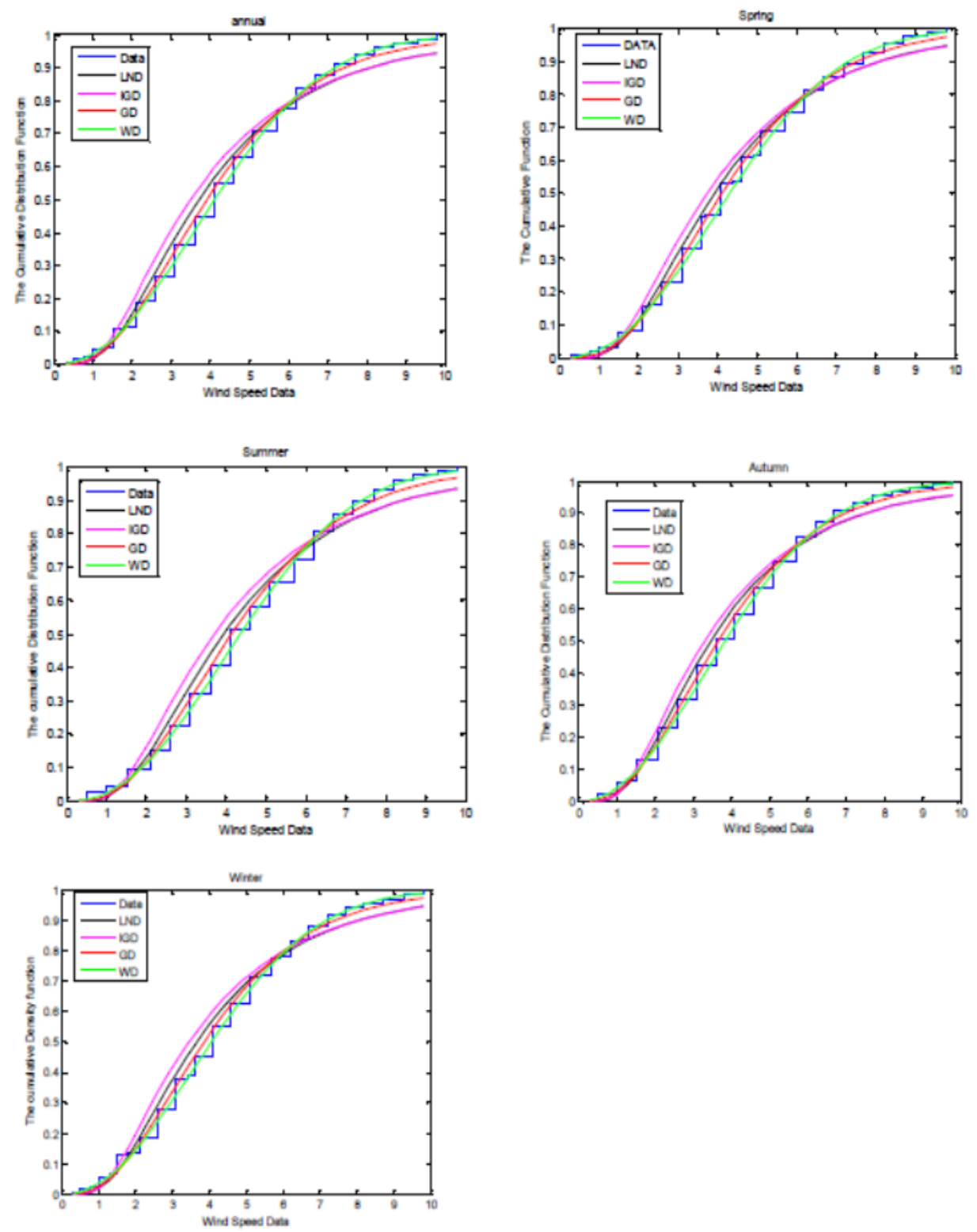

Figure 1. The cumulative density function for annual and seasonal wind speed data $(m / s)$ for Sivas. 
Table 6. Classical parameter estimations for the wind speed data.

\begin{tabular}{|c|c|c|c|c|}
\hline $\mathrm{n}$ & & Estimator & $\hat{\alpha}$ & $\hat{\beta}$ \\
\hline & & MLE & 2.1520 & 4.9177 \\
\hline & & LME & 2.1422 & 4.9202 \\
\hline & & TLME & 2.1256 & 4.9326 \\
\hline & & MMLE-I & 2.1318 & 4.9088 \\
\hline 6011 & Annual & MMLE-II & 3.0342 & 5.2794 \\
\hline & & $\mathrm{ME}$ & 2.1551 & 4.9203 \\
\hline & & LSE & 2.1083 & 4.9360 \\
\hline & & WLSE & 2.1571 & 4.9221 \\
\hline & & $\mathrm{PE}$ & 2.1876 & 4.9300 \\
\hline & & MLE & 2.1052 & 4.8413 \\
\hline & & LME & 2.1020 & 4.8463 \\
\hline & & TLME & 2.0534 & 4.8832 \\
\hline & & MMLE-I & 2.0780 & 4.8291 \\
\hline 1560 & Winter & MMLE-II & 2.6032 & 5.0557 \\
\hline & & $\mathrm{ME}$ & 2.1139 & 4.8465 \\
\hline & & LSE & 2.1089 & 4.8786 \\
\hline & & WLSE & 2.1168 & 4.8610 \\
\hline & & $\mathrm{PE}$ & 2.1452 & 4.8600 \\
\hline & & MLE & 2.2734 & 5.0726 \\
\hline & & LME & 2.2605 & 5.0705 \\
\hline & & TLME & 2.3060 & 5.0389 \\
\hline & & MMLE-I & 2.2730 & 5.0724 \\
\hline 1710 & Spring & MMLE-II & 2.6083 & 5.2082 \\
\hline & & $\mathrm{ME}$ & 2.2674 & 5.0704 \\
\hline & & LSE & 2.1956 & 5.0535 \\
\hline & & WLSE & 2.2540 & 5.0615 \\
\hline & & $\mathrm{PE}$ & 2.2746 & 5.0723 \\
\hline & & MLE & 2.2483 & 5.1749 \\
\hline & & LME & 2.2290 & 5.1816 \\
\hline & & TLME & 2.1682 & 5.2274 \\
\hline & & MMLE-I & 2.2058 & 5.1573 \\
\hline 1429 & Summer & MMLE-II & 2.5317 & 5.2892 \\
\hline & & $\mathrm{ME}$ & 2.2541 & 5.1812 \\
\hline & & LSE & 2.1641 & 5.2318 \\
\hline & & WLSE & 2.2420 & 5.1998 \\
\hline & & $\mathrm{PE}$ & 2.3019 & 5.1970 \\
\hline
\end{tabular}


Table 6. Continued.

\begin{tabular}{|c|c|c|c|c|}
\hline \multirow{2}{*}{\multicolumn{2}{|c|}{$\mathrm{n}$}} & Estimator & $\hat{\alpha}$ & $\hat{\beta}$ \\
\hline & & MLE & 2.0056 & 4.5226 \\
\hline \multirow{8}{*}{1312} & & LME & 2.0020 & 4.5226 \\
\hline & & TLME & 2.0117 & 4.5155 \\
\hline & & MMLE-I & 1.9993 & 4.5196 \\
\hline & Autumn & MMLE-II & 2.5919 & 4.7879 \\
\hline & & $\mathrm{ME}$ & 2.0060 & 4.5228 \\
\hline & & LSE & 1.9794 & 4.5225 \\
\hline & & WLSE & 2.0062 & 4.5257 \\
\hline & & $\mathrm{PE}$ & 2.0140 & 4.5285 \\
\hline
\end{tabular}

Table 7.Lindley's and Tierney Kadane's parameter estimations under NP for the wind speed data

\begin{tabular}{|c|c|c|c|c|c|c|}
\hline \multirow[b]{2}{*}{$\mathrm{n}$} & & \multirow[b]{2}{*}{ LF } & \multicolumn{2}{|c|}{ Lindley's } & \multicolumn{2}{|c|}{ Tierney-Kadane's } \\
\hline & & & $\hat{\alpha}$ & $\hat{\beta}$ & $\hat{\alpha}$ & $\hat{\beta}$ \\
\hline \multirow{4}{*}{6011} & \multirow{4}{*}{ Annual } & SELF & 2.1517 & 4.9178 & 2.1494 & 4.9178 \\
\hline & & GELF & 2.1515 & 4.9176 & 2.1493 & 4.9177 \\
\hline & & WSELF & 2.1515 & 4.9176 & 2.1492 & 4.9176 \\
\hline & & PLF & 2.1518 & 4.9179 & 2.1496 & 4.9179 \\
\hline \multirow{4}{*}{1560} & \multirow{4}{*}{ Winter } & SELF & 2.1041 & 4.8419 & 2.0963 & 4.8418 \\
\hline & & GELF & 2.1034 & 4.8412 & 2.0956 & 4.8414 \\
\hline & & WSELF & 2.1033 & 4.8411 & 2.0954 & 4.8413 \\
\hline & & PLF & 2.1046 & 4.8423 & 2.0967 & 4.8423 \\
\hline \multirow{4}{*}{1710} & \multirow{4}{*}{ Spring } & SELF & 2.2724 & 5.0731 & 2.2619 & 5.0731 \\
\hline & & GELF & 2.2717 & 5.0725 & 2.2611 & 5.0726 \\
\hline & & WSELF & 2.2716 & 5.0724 & 2.2611 & 5.0724 \\
\hline & & PLF & 2.2728 & 5.0734 & 2.2624 & 5.0734 \\
\hline \multirow{4}{*}{1429} & \multirow{4}{*}{ Summer } & SELF & 2.2471 & 5.1755 & 2.2349 & 5.1753 \\
\hline & & GELF & 2.2463 & 5.1748 & 2.2340 & 5.1749 \\
\hline & & WSELF & 2.2462 & 5.1747 & 2.2338 & 5.1748 \\
\hline & & PLF & 2.2476 & 5.1759 & 2.2355 & 5.1758 \\
\hline \multirow{4}{*}{1312} & \multirow{4}{*}{ Autumn } & SELF & 2.0044 & 4.5232 & 1.9973 & 4.5233 \\
\hline & & GELF & 2.0036 & 4.5224 & 1.9965 & 4.5223 \\
\hline & & WSELF & 2.0035 & 4.5223 & 1.9963 & 4.5222 \\
\hline & & PLF & 2.0049 & 4.5237 & 1.9978 & 4.5237 \\
\hline
\end{tabular}


Table 8. Lindley's and Tierney Kadane's parameter estimations under GP for the wind speed

\begin{tabular}{|c|c|c|c|c|c|c|}
\hline & & & \multicolumn{2}{|c|}{ Lindley's } & \multicolumn{2}{|c|}{ Tierney-Kadane's } \\
\hline $\mathrm{n}$ & & $\mathrm{LF}$ & $\hat{\alpha}$ & $\hat{\beta}$ & $\hat{\alpha}$ & $\hat{\beta}$ \\
\hline \multirow{4}{*}{6011} & \multirow{4}{*}{ Annual } & SELF & 2.1517 & 4.9186 & 2.1489 & 4.918 \\
\hline & & GELF & 2.1515 & 4.9184 & 2.1487 & 4.9131 \\
\hline & & WSELF & 2.1515 & 4.9184 & 2.1487 & 4.9131 \\
\hline & & PLF & 2.1518 & 4.9187 & 2.1490 & 4.9187 \\
\hline \multirow{4}{*}{1560} & \multirow{4}{*}{ Winter } & SELF & 2.1039 & 4.8365 & 2.0939 & 4.8365 \\
\hline & & GELF & 2.1034 & 4.8358 & 2.0953 & 4.8358 \\
\hline & & WSELF & 2.1033 & 4.8357 & 2.0951 & 4.8357 \\
\hline & & PLF & 2.1046 & 4.8369 & 2.0965 & 4.8369 \\
\hline \multirow{4}{*}{1710} & \multirow{4}{*}{ Spring } & SELF & 2.2727 & 5.0731 & 2.2623 & 5.0731 \\
\hline & & GELF & 2.2720 & 5.0725 & 2.2615 & 5.0725 \\
\hline & & WSELF & 2.2719 & 5.0725 & 2.2614 & 5.0725 \\
\hline & & PLF & 2.2731 & 5.0734 & 2.2627 & 5.0734 \\
\hline \multirow{4}{*}{1429} & \multirow{4}{*}{ Summer } & SELF & 2.2494 & 5.1776 & 2.2371 & 5.1777 \\
\hline & & GELF & 2.2486 & 5.1769 & 2.2361 & 5.1770 \\
\hline & & WSELF & 2.2484 & 5.1768 & 2.2360 & 5.1769 \\
\hline & & PLF & 2.2499 & 5.1780 & 2.2376 & 5.1781 \\
\hline \multirow{4}{*}{1312} & \multirow{4}{*}{ Autumn } & SELF & 2.0059 & 4.5218 & 1.9984 & 4.5218 \\
\hline & & GELF & 2.0047 & 4.5210 & 1.9976 & 4.5210 \\
\hline & & WSELF & 2.0046 & 4.5209 & 1.9975 & 4.5209 \\
\hline & & PLF & 2.0060 & 4.5223 & 1.9989 & 4.5223 \\
\hline
\end{tabular}

It is clear that the results in Figure 1 are consistent with Table 4. Thus, in terms of all criteria, WD performed better than GD, IGD and LND for the seasonal and the annual wind speed data. Therefore, the two-parameter Weibull distribution was used for modelling the wind speed data. The estimators of the $\alpha$ and $\beta$ obtained by using Bayesian and classical methods are given in Table 6-8. In light of the aforementioned information, we recommend the Bayesian estimations under WSELF and GELF for estimating the unknown parameters of Weibull distribution.

\section{Conclusion}

In this paper, we obtained different methods of estimation of the unknown parameters both with Bayesian and classical approximation. In classical method, the parameters $\alpha$ and $\beta$ were estimated by using nine different method. In Bayesian method, we computed the Bayesian estimators of unknown parameters based on symmetric and asymmetric loss functions. The Bayes estimators do not have explicit form. Hence, we used the Lindley and Tierney Kadane's techniques under 
the assumption of Gamma priors. We also compare the performances of the estimators via simulation study. It is clear from the simulation results given in Table 1-3 that Lindley approximation under GELF and WSELF are more preferable than the other estimators according to the MSE and bias criteria in both scenarios i.e. informative prior and non-informative prior (especially for sample size $n>50$ ).

\section{REFERENCES}

[1] Lawless, J. F., Statistical Models and Methods for Life time Data, 3rd Edition, John Wiley and Sons, New York 2003

[2] Tang, Y., Extended Weibull Distributions in Reliability Engineering, A Thesis Submitted to Department of Industrial System Engineering, National University of Singapore, 2004.

[3] Justus, C. G., et al., Methods for estimating wind speed frequency distributions, Journal of applied meteorology, 17(3), (1978) 350-353.

[4] Kantar, Y. M., Şenog̃lu, B., (2008). A comparative study for the location and scale parameters of the Weibull distribution with given shape parameter, Computers Geosciences, 34(12), (2008), 1900-1909.

[5] Genc, A., et al. Estimation of wind power potential using Weibull distribution, Energy Sources, 27(9), (2005), 809-822.

[6] Stevens, M. J. M., and Smulders, P. T., The estimation of the parameters of the Weibull wind speed distribution for wind energy utilization purposes, Wind engineering, (1979), 132-145.

[7] Araki, Junichi, et al., Weibull distribution function for cardiac contraction: integrative analysis, American Journal of Physiology-Heart and Circulatory Physiology, 277(5), (1999), 19401945.

[8] Fernandez, A., et al., Application of a frequency distribution model to describe the thermal inactivation of two strains of Bacillus cereus, Trends in food science technology, 10(4), (1999), 158-162.

[9] Zobeck, Ted M., Thomas, E. Gill, and Thomas, W. Popham, A two-parameter Weibull function to describe airborne dust particle size distributions, Earth Surface Processes and Landforms: The Journal of the British Geomorphological Research Group, 24(10), (1999), 943-955.

[10] Trustrum, K., Jayatilaka, A. S., On estimating the Weibull modulus for a brittle material, Journal of Material Science, 14, (1979),1080-1084.

[11] Hung, Wen-Liang, Weighted least-squares estimation of the shape parameter of the Weibull distribution, Quality and Reliability Engineering International, 17(6 ) (2001), 467-469.

[12] Lu, Hai-Lin, Chen, Chong-Hong and Wu, Jong-Wuu, A note on weighted least-squares estimation of the shape parameter of the Weibull distribution. Quality and Reliability Engineering International, 20.6 (2004), 579-586.

[13] Pobocâkova, Ivana, and Sedliackova, Zuzana, Comparison of four methods for estimating the Weibull distribution parameters, Appl. Math. Sci. 8(1), (2014), 4137-4149.

[14] Teimouri, Mahdi, Hoseini, Seyed M. and Nadarajah, Saralees, Comparison of estimation methods for the Weibull distribution. Statistics 47(1) (2013), 93-109.

[15] Alizadeh, M., Rezaei, S. and Bagheri, S. F., On the estimation for the Weibull distribution, Annals of Data Science, 2(4) (2015), 373-390.

[16] Al Omari, M.A. and Ibrahim, N.A., Bayesian survival estimator for Weibull distribution with censored Data, J. Appl. Sci. 11, (2011), 393-396.

[17] Guure, Chris Bambey, Ibrahim, Noor Akma and Al Omari, Mohammed Ahmed, Bayesian estimation of two-parameter weibull distribution using extension of Jeffreys' prior information with three loss functions, Mathematical Problems in Engineering 2012 (2012). 
[18] Pandey, B.N., Dwividi, N. and Pulastya, B., Comparison between Bayesian and maximum likelihood estimation of the scale parameter in Weibull distribution with known shape under linex loss function, J. Sci. Res., 55, (2011), 163-172.

[19] Al-Athari, F.M., Parameter estimation for the double-Pareto distribution, J. Mathematics Statistics, 7 (2011), 289-294.

[20] Zellner, Arnold, Bayesian estimation and prediction using asymmetric loss functions, Journal of the American Statistical Association, 81(394), (1986), 446-451.

[21] Kundu, D. and Raqab, M.Z., Generalized Rayleigh distribution: Different methods of estimations, Comput. Statist.Data Anal., 49 (2005), 187-200.

[22] Ramos, Pedro Luiz, et al., The Frechet distribution: Estimation and Application an Overview, arXiv preprint arXiv 2018:1801.05327.

[23] Dey, Sanku, et al., Two-parameter Maxwell distribution: Properties and different methods of estimation, Journal of Statistical Theory and Practice, 10(2) (2016), 291-310.

[24] Alkasasbeh, M.R. and Raqab, M.Z., Estimation of the generalized logistic distribution parameters: Comparative study, Statist. Methodol. 6, (2009), 262-279.

[25] Howard, R., Antle, C. and Klimko, L.A., Maximum likelihood estimation with the Weibull model, Journal of the American Statistical Association, 69(345) (1974), 246-249.

[26] Cohen, A.C., Maximum likelihood estimation in the Weibull distribution based on complete and on censored samples, Technometrics, 7(4) (1965), 579-588.

[27] Swain, James J., Venkatraman, Sekhar and Wilson, James R., Least-squares estimation of distribution functions in Johnson's translation system, Journal of Statistical Computation and Simulation 29(4) (1988), 271-297.

[28] Kao, John H.K., Computer methods for estimating Weibull parameters in reliability studies, IRE Transactions on Reliability and Quality Control, (1958), 15-22.

[29] Kao, John H.K., A graphical estimation of mixed Weibull parameters in life-testing of electron tubes, Technometrics, 1(4) (1959), 389-407.

[30] Hosking, Jonathan R.M, L-moments: Analysis and estimation of distributions using linear combinations of order statistics, Journal of the Royal Statistical Society: Series B (Methodological) 52(1) (1990), 105-124.

[31] Abdul-Moniem, I. B., TL-moments and L-moments estimation for the Weibull distribution, Advances and Application in Statistics, $15(1$ ) (2009), 83-99.

[32] Elamir, Elsayed A.H, and Seheult, Allan H, Trimmed L-moments, Computational Statistics Data Analysis, 43(3 ) (2003), 299-314.

[33] Cohen, C.A., and Whitten, B., Modified maximum likelihood and modified moment estimators for the three-parameter Weibull distribution. Communications in Statistics-Theory and Methods, 11(23) (1982), 2631-2656.

[34] Box Cep, Tiag, G. C., Bayesian inference in statistical analysis, 1973.

[35] Renjini, K. R., Abdul-Sathar, E. I. and Rajesh, G. A study of the effect of loss functions on the Bayes estimates of dynamic cumulative residual entropy for Pareto distribution under upper record values, Journal of Statistical Computation and Simulation, 86(2) (2016), 324-339.

[36] Ali, Sajid, Aslam, Muhammad and Kazmi, Syed Mohsin Ali, A study of the effect of the loss function on Bayes Estimate, posterior risk and hazard function for Lindley distribution, Applied Mathematical Modelling, 37(8), (2013), 6068-6078.

[37] Legendre, A., New Methods for the Determination of Orbits of Comets Courcier, Paris, 1805.

[38] Gauss, C. F., Least squares method for the combinations of observations (translated by J. Bertrand 1955), Mallet-Bachelier, Paris, 1810.

[39] Feynman, Richard P., Mr. Feynman goes to Washington, Engineering and Science, 51(1), (1987), 6-22.

[40] Calabria, R. and Pulcini, G., An engineering approach to Bayes estimation for the Weibull distribution, Micro Electron Reliab, 34, (1994), 789-802. 
[41] Norstrom, Jan Gerhard, The use of precautionary loss functions in risk analysis, IEEE Transactions on reliability, 45(3), (1996), 400-403.

[42] Helu, Amal and Samawi, Hani, The Inverse Weibull Distribution as a Failure Model Under Various Loss Functions and Based on Progressive First-Failure Censored Data, Quality Technology Quantitative Management, 12(4), (2015), 517-535.

[43] Ali, Sajid, On the mean residual life function and stress and strength analysis under different loss function for Lindley distribution, Journal of Quality and Reliability Engineering, 2013.

[44] O'Hagan, A., and Luce, B. R., A primer on Bayesian statistics in health economics and outcomes research, Sheffield: Centre for Bayesian Statistics in Health Economics, 2003.

[45] Acquah, H. D., Bayesian Logistic Regression Modelling via Markov Chain Monte Carlo Algorithm, Journal of Social and Development Sciences, 4(4), (2013), 193-197.

[46] Lindley, Dennis V., Approximate Bayesian methods. Trabajos de estadstica y de investigacin operativa, 31(1), (1980), 223-245.

[47] Tierney, Luke, and Kadane, Joseph B., Accurate approximations for posterior moments and marginal densities, Journal of the American statistical association, 81(393), (1986), 82-86.

[48] Singh, S. K., Singh, U. and Yadav, A. S. Bayesian estimation of Marshall-Olkin extended exponential parameters under various approximation techniques, Hacettepe Journal of Mathematics and Statistics, 43(2), (2014), 347-360.

Current address: Asuman Yilmaz: Van Yuzuncu Yil University, Faculty of Economis and Administrative Sciences, Depatment of Econometrics, 65080 Van, Tutkey.

E-mail address: asumanduva@yyu.edu.tr

ORCID Address: https://orcid.org/0000-0002-8653-6900

Current address: Mahmut Kara: Van Yuzuncu Yil university, Faculty of Economis and Administrative Sciences, Depatment of Econometrics, 65080 Van, Tutkey.

E-mail address: mkara2581@gmail.com

ORCID Address: https://orcid.org/0000-0001-7678-8824

Current address: Halil Aydogdu: Ankara University, Faculty of sciences, Department of Statistics, 06100, Tandogan, Ankara.

E-mail address: aydogdu@ankara.edu.tr

ORCID Address: https://orcid.org/0000-0001-5337-5277 